\title{
ARANEOFAUNA URBANA (ARACHNIDA: ARANEAE) DE CIUDAD VICTORIA, TAMAULIPAS, MÉXICO
}

\author{
Carlos A. SALAZAR-OLIVO ${ }^{1}$ \& CARLos SOLÍS-ROJAS ${ }^{2}$
}

\author{
${ }^{1}$ Laboratorio de Ecología, ITCV. Portes Gil 1301 Pte., Ciudad Victoria, Tamps. <salazar.olivo@gmail.com> \\ ${ }^{2}$ Laboratorio de Arthropoda, FCB UANL. Ciudad Universitaria, N. L. <csolis01@gmail.com> \\ Recibido: 24/03/2014; aceptado: 29/10/2014
}

\begin{abstract}
Salazar-Olivo, C. A. \& Solís-Rojas, C. 2015. Araneofauna urbana (Arachnida: Araneae) de Ciudad Victoria, Tamaulipas, México. Acta Zoológica Mexicana (n. s.), 31(1): 55-66.
\end{abstract}

RESUMEN. Se describe la araneofauna urbana en el área de Ciudad Victoria, Tamaulipas, México y su distribución sobre la mancha urbana. Se realizó un muestreo por conglomerados en tres etapas y se identificaron 59 especies correspondientes a 29 familias. Se encontraron dos nuevos registros para el estado y se presentan los valores de riqueza específica, diversidad y equitatividad, así como los valores de sinantropía asociados a las especies. Además se analizó la similitud entre cinco zonas de la ciudad.

Palabras clave: Fauna Urbana, Sinantropía, Araneofauna.

\section{INTRODUCCIÓN}

Históricamente, las arañas han convivido con los humanos en sus asentamientos, probablemente desde que se fundaron las primeras aldeas; basta recordar el mito de Arachne en las Metamorfosis de Ovidio, o las alusiones a los tipos de araña "Tocatl”, que conocían los aztecas, según el Código Florentino. Actualmente, el conocimiento sobre la araneofauna en zonas urbanas está tomando mayor interés, sobre todo considerando la presencia de especies peligrosas para los habitantes de las ciudades (Quintana-Castillo \& Otero-Patiño 2002; Borda et al. 2005), y al hecho de que la urbanización puede modificar las poblaciones de artrópodos originales de la zona (McIntyre 2000).

En México, la investigación sobre la fauna de arañas urbanas se inicia a fines del siglo anterior, con los trabajos de Jiménez (1998), que estudió las arañas de las viviendas en la ciudad de la Paz, B. C. S. y Castelo y Pérez (1999), que señala las dificultades en la determinación de algunos géneros de salticidos sinantrópicos de la Ciudad de México. En la Ciudad de México, Durán-Barron ha estudiado la araneofauna urbana (Durán-Barrón \& Pérez-Ortiz 2005; Durán-Barrón 2007; Durán-Barrón et al. 2009), mientras que Desales-Lara et al. en 2011 y 2013, revisan las arañas sinantrópicas en la Ciudad de Toluca, Mex. Existen además varios tratados sobre artrópodos en general asociados a zonas urbanas como se observa en el estudio elaborado por Cupul-Magaña y Navarrete-Heredia (2008), en Puer-
Salazar-Olivo, C. A. \& Solís-Rojas, C. 2015. Urban spider fauna (Arachnida: Araneae) in Ciudad Victoria, Tamaulipas, Mexico. Acta Zoológica Mexicana (n. s.), 31(1): 55-66.

ABSTRACT. We describe urban spider faun in Ciudad Victoria, Tamaulipas, Mexico and it's distribution on the urban area. Cluster sampling was performed in three steps and 59 species were identified corresponding to 29 families. Two new records for the state were found, and the values for species richness, diversity and evenness, also level of synanthropy associated with the species are presented. Similarity was analyzed from five areas of the city.

Key words: Urban Fauna, Synanthropy, Spider Fauna.

to Vallarta, Jal. Estos trabajos, aparte de contribuir al conocimiento de la fauna sinantrópica, permite determinar su distribución y el descubrimiento de nuevas especies, como en el trabajo de Desales-Lara (2012).

La araneofauna de la región central de Tamaulipas y más concretamente de Ciudad Victoria está pobremente conocida, aún y cuando existen varios artículos realizados por investigadores extranjeros. Las obras con mayor énfasis en especies tamaulipecas son de Gertsch y Davis (1937), Chickering (1937), Reddell y Elliott (1973) y Gómez-Rodríguez y Salazar-Olivo (2012). Gómez-Rodríguez et al. (en prensa), elaboraron un listado actualizado de arácnidos para el Estado de Tamaulipas anotando 437 especies de arácnidos excepto ácaros, de los cuales 350 son Araneae. En 1976, Hoffman reporta 215 especies de arañas para el estado, y Jiménez (1996) amplía ese número a 227. Ruiz-Cancino y Coronado-Blanco (2002), revisando la literatura para el estado, reportan un total de 135 especies para los Estados de Nuevo León y Tamaulipas, en donde 65 especies corresponden a Tamaulipas.

Este trabajo describe la araneofauna colectada en la zona urbana y suburbana de Ciudad Victoria, Tamaulipas. y busca incrementar el conocimiento del estado actual de la fauna de arañas del estado.

\section{MATERIAL Y MÉTODOS}

Área de estudio. Ciudad Victoria está situada al oriente de la Sierra Madre Oriental, en la porción centro del 
estado (Fig. 1), en las coordenadas $23^{\circ} 43^{\prime}$ latitud N. y 99 ${ }^{\circ} 7^{\prime}$ longitud O. a una altitud de 318 msnm, y cuenta con una superficie de $188 \mathrm{~km}^{2}$, con poco más de $320 \mathrm{mil}$ habitantes (INEGI, 2012). La vegetación nativa del municipio que rodea la mancha urbana, la constituyen matorrales altos y medianos de 3 a $5 \mathrm{~m}$, de tipo subinermes y espinosos, y matorrales inermes parvifolios. Los componentes predominantes son la barreta (Helietta parvifolia), zapotillo (Bourreria), laurelillo (Quercus laurina), anacahuita (Cordia boissieri), ébano (Phytecellobium ebano), mezquite (Prosopis glandulosa), chaparro prieto (Vachellia rigidula), tenaza (Pithecellobium pallens), cenizo (Leucophyllum frutescens), granjeno (Celtis palli$d a$ ), etc. Dentro de las variedades de vegetación inducida destacan el cultivo de cítricos (limón, naranja y pomelo) y árboles ornamentales como la Jacaranda (Jacaranda), Framboyán (Delonix), Álamo (Populus), Fresno (Fraxinus) y Olmo (Ulmus) (Anónimo, 1983). Los suelos son sedimentarios, arcillosos y arenosos (Anónimo, 1983). El clima, según la clasificación Köppen-García, en la porción del plano inclinado del Municipio que es donde se asienta la ciudad, es BS1(h')w”(e) de tipo estepario en un 15\% semicálido-semiseco, siendo los meses de mayor precipitación Junio, Agosto, Septiembre y Octubre, y los de menor Enero, Marzo y Diciembre; con una precipitación promedio anual, de 924mm. (Anónimo 1983).

Método. Se dividió el área urbana en 5 zonas (fig 1); una zona central y cuatro periféricas, y se procedió a subdividir estas zonas en manzanas como segundo nivel y casas habitación como tercer nivel. Las manzanas fueron escogidas al azar, utilizando un generador de números aleatorios, y las casas de estas manzanas se seleccionaron por el criterio de respuesta de sus habitantes (permiso de los moradores de realizar el muestreo). Para la búsqueda de arañas, se realizaron tres muestreos por domicilio en un total de 50 casas, durante el año 2012, con una periodicidad de dos meses. El primer muestreo inició a principios de abril y finalizó a mediados de mayo; el segundo comenzó al inicio de junio y finalizó a mediados de julio y a principios de agosto se inició el último, finalizando a mediados de septiembre. De las casas revisadas, el total (50) eran de material de block o ladrillo con al menos zar-

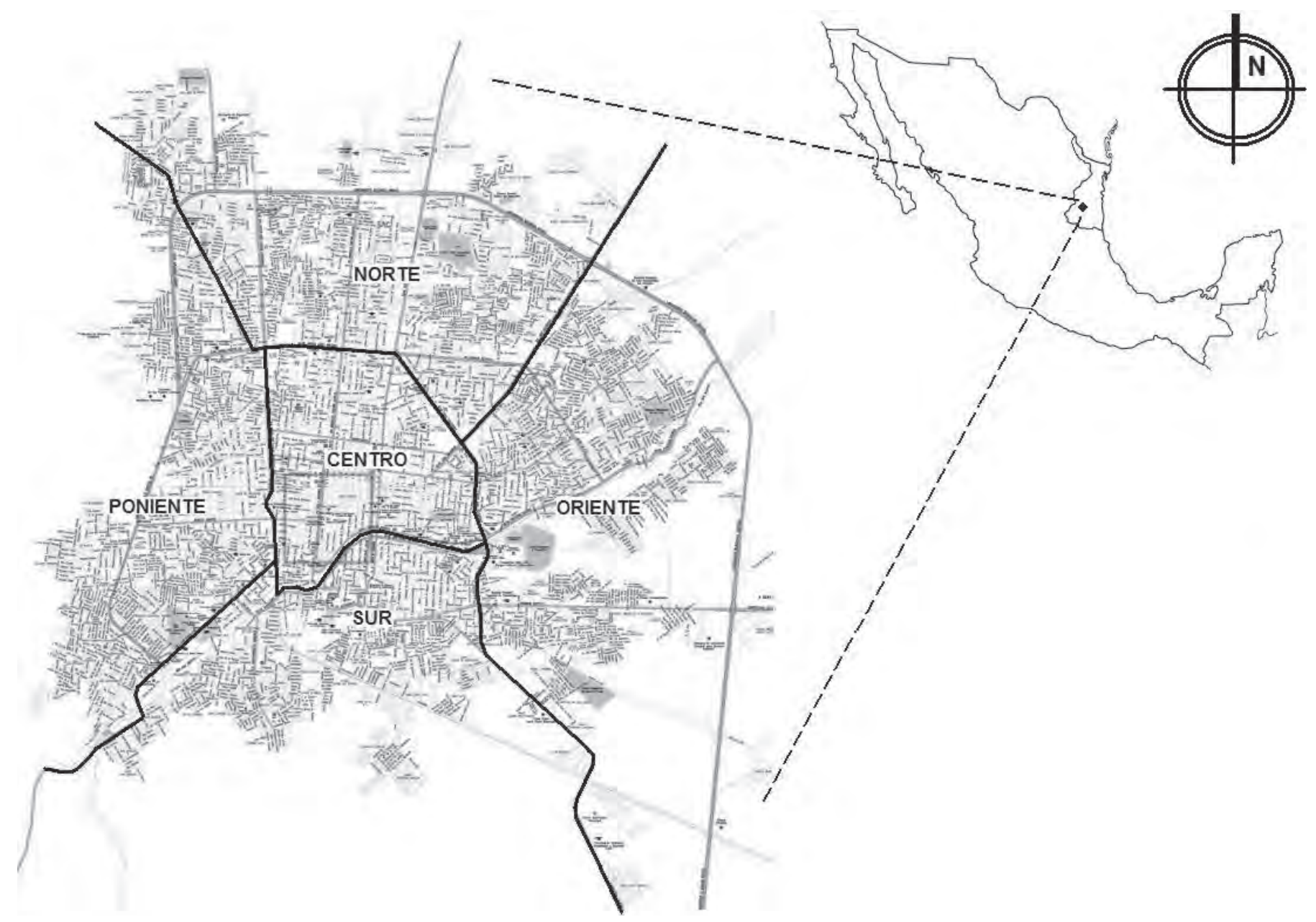

Figura 1. Localización del área de estudio. Tomado y modificado de INEGI, Dirección Regional Noreste. 
peo interior y piso de cemento; el 18\% eran casas en obra negra y las 41 restantes (82\%) presentaban acabado como yeso o estuco en paredes, y pisos de mosaico o azulejo. El 74\% presentaba arboles en las aceras, principalmente albizias (Pithecellobium dulce), truenos (Ligustrum japonicum), framboyanes (Delonix regia), jacarandas (Jacaranda sp), mezquite (Prosopis glandulosa), y pata de vaca (Bauhinia macrathera) o arbustos (principalmente $\mathrm{Ca}$ tharanthus roseus); el 46\% presentaba algún otro tipo de vegetación en el exterior (jardines o porche con plantas en macetas).

Los muestreos se realizaron sábados y domingos, entre las 10 y las 18 horas, trabajando en 5 ó 6 casas por día de trabajo. Cada muestreo se llevó a cabo en el área común de la casa (sala-comedor, baño y cocina) y en el área externa (banqueta, cochera, jardín o porche). El muestreo consistió en una revisión a fondo del interior del domicilio, examinando bajo muebles, tras cuadros, en rincones, tras puertas y ventanas, etc. En el exterior, se revisaron plantas y macetas, el jardín, en caso de existir, material diverso acumulado en los porches y vegetación en el área circundante a la vivienda, hasta la acera. Se colectó el material biológico utilizando pinzas de disección y aspirador entomológico y sacrificio y conservación de los ejemplares en alcohol etílico al 96\%. Se colectó en cada casa durante aproximadamente una hora por dos personas, una en el interior y otra en el exterior. En total se obtuvieron 150 muestras, en un período de 6 meses.Todo el material colectado se conserva en la Colección de Arácnidos del Instituto Tecnológico de Ciudad Victoria (ITCV).

Para la determinación taxonómica de los especímenes colectados se utilizaron las obras de Bradley (2013), Jocqué y Dippenaar-Schoeman (2006), Ubick et al. (2005) y Kaston (1978). Se revisó el Catálogo Mundial de Arañas de Platnick (2014), para corroborar la distribución y sinonimia de la especie. Para géneros y especies, se utlizaron los trabajos de Brady (1964), Levi y Randolph (1975), Gertsch y Davis (1937, 1940, 1940, 1942, 1946), Gertsch y Mulaik (1940), Gertsch y Ennik (1983), Huber (2000), Levi (2002), y Prószyński (2013). Para algunas hembras, se realizaron disecciones de genitalia, según las técnicas de Levi (1965) y para machos, se observaron los palpos en preparaciones temporales en portaobjetos excavados.
Para caracterizar la comunidad de arañas, se separaron los ejemplares colectados en el interior de las viviendas y en la periferia, así como en las cinco zonas en las que se dividió la ciudad, y se calcularon los índices de diversidad de Shannon-Weaver $\left(\mathrm{H}^{\prime}\right)$ y la equitatividad de Pielou (J) según los criterios de Brower y Zar (1977). Se calcularon, además, los intervalos de confianza para la diversidad (Pla 2006), y para comparar gráficamente las diversidades entre zonas y entre interior y exterior, se utilizó la transformación de orden $q=1$, según el criterio de Jost (2006). La diversidad de Shannon-Weaver se calculó mediante $\mathrm{H}=-\sum_{\mathrm{i}=1}^{\mathrm{S}} \mathrm{p}_{\mathrm{i}} \ln \mathrm{p}_{\mathrm{i}}$, con $\mathrm{p}_{\mathrm{i}}=\mathrm{n}_{\mathrm{i}} / \mathrm{N}, \mathrm{n}_{\mathrm{i}}=$ número de individuos de la especie i en la muestra y $\mathrm{N}=$ el total de la muestra; y la equitatividad de Pielou como $\mathrm{J}=\mathrm{H} / \mathrm{H}_{\max }$ donde $\mathrm{H}$ es la Diversidad de Shannon-Weaver, $\mathrm{y} \mathrm{H}_{\max }=\ln \mathrm{S}$. Para los intervalos de confianza de la diversidad de Shannon-Weaver se utilizó la fórmula $\operatorname{Var}(\mathrm{H})=\frac{1}{\mathrm{~N}}\left[\sum_{\mathrm{i}=1}^{\mathrm{S}} \mathrm{Pi}\left(\operatorname{lnp}_{\mathrm{i}}\right)^{2}-\mathrm{H}^{2}\right]+\frac{\mathrm{S}-1}{2 \mathrm{~N}^{2}}$. La diversidad de orden q se obtuvo mediante ${ }^{\mathrm{q}} \mathrm{D} \equiv\left(\sum_{\mathrm{i}=1}^{\mathrm{s}} \mathrm{p}_{\mathrm{i}}^{\mathrm{q}}\right)^{1 /(1-\mathrm{q})}$ y para el caso de $\mathrm{q}=1,{ }^{1} \mathrm{D}=\mathrm{e}^{\mathrm{H}}$.

Para la riqueza específica, se elaboraron curvas de acumulación de especies que relacionan la aparición de especies en la muestra con el esfuerzo de muestreo, según la metodología descrita por Jiménez-Valverde y Hortal (2003); las curvas se ajustaron al modelo de Clench (Soberon \& Llorente 1993), dado que las casas (el ambiente) son muy heterogeneneas y existe un alto número de especies raras (con poca abundancia), mediante el programa EstimateS (Colkwell 2013). Se utilizó la razón a/b de la ecuación de Clench, para calcular la riqueza específica (Jimenez-Valverde \& Hortal 2003).

Con el fin de determinar las especies sinantrópicas, se calcularon los índices de infestación (II) y los índices de densidad (ID) de García, modificados por Jiménez (1998), y el índice de permanencia propuesto por Durán-Barrón et al. (2009). La metodología y los criterios para categorizar el estatus sinantrópico de cada especie se tomó de Desales-Lara et al. (2013), quien resumió los intervalos propuestos por Durán-Barrón et al. (2009), y se muestran en el cuadro 1. La sinantropía se complementó utilizando

Cuadro 1. Niveles de Sinantropía basados en el índice de Densidad (ID), Índice de Infestación (II) e Índice de Permanencia (IP). Tomados de Desales-Lara et al. 2013.

\begin{tabular}{llll}
\hline Nivel de sinantropía & Intervalos ID & Intervalos I I & Intervalos I P \\
\hline Accidentales & $0-0.9$ & $0-1$ & $1-10$ \\
Ocasionales & $1-2.9$ & $1.1-3.5$ & $11-30$ \\
Frecuentes & $3.0-9.9$ & $3.6-9.9$ & $31-50$ \\
Comunes & 10 - en adelante & 10 - en adelante & 51 - en adelante \\
\hline
\end{tabular}


la modificación a la prueba de Olmstead-Tukey (O-T), propuesta por Desales-Lara et al. (2013). Las fórmulas utilizadas fueron: índice de densidad (ID) $)_{\mathrm{i}}=$ (número de arañas de la especie i / número de viviendas donde se localizó la especie i)*100, índice de infestación (II) ${ }_{\mathrm{i}}=$ (número de viviendas donde se localizó la especie i / número de viviendas revisadas)*100, e índice de permanencia $(\mathrm{IP})_{\mathrm{i}}=$ (número de meses en que se colectó la araña i / número de meses totales)*100.

Para la prueba O-T, se graficó en un plano cartesiano para cada especie, el logaritmo de su abundancia en las abscisas y en la ordenada, el número de localidades donde se localizó la especie; el plano se subdivide en cuatro regiones mediante la mediana de los rangos, lo que permite generar cuadrantes relativos a la categoría de sinantropismo de la especie. La posición relativa de cada especie en los cuadrantes, señala su nivel de sinantropía; el cuadrante superior derecho corresponde a las especies "sinantrópicas comunes", el inferior derecho las especies sinantrópicas frecuentes" y el cuadrante inferior izquierdo, las especies accidentales.

Finalmente, se comparó la composición de especies entre zonas de la ciudad, para especies localizadas en el interior de las viviendas y para la periferia, mediante el coeficiente de similitud de Jaccard para presencia y ausencia. El coeficiente de similitud de Jaccard, se calculó como $\mathrm{J}=\frac{\mathrm{c}}{\mathrm{a}+\mathrm{b}-\mathrm{c}}$ donde $\mathrm{a}=$ número de especies en el sitio $\mathrm{A}$, $\mathrm{b}$ = número de especies del sitio $\mathrm{B}$ y c = número de especies comunes en ambos sitios (Moreno 2001, Magurran 2004). Los valores de similitud se utilizaron para la elaboración de un diagrama de similaridad entre zonas, mediante el método UPGMA, de distancia promedio entre grupos (Sneath \& Sokal 1973).

\section{RESULTADOS}

Se identificaron 893 ejemplares pertenecientes a 29 familias, 56 géneros y 59 especies (Cuadros 3 y 4). Se colectaron 30 especies del interior de las casas y 37 en la periferia. La especie mas abundante en el interior de los domicilios (Cuadro 3), fué Psilochorus redemptus (Pholcidae), seguida de Kukulkania hibernalis (Filistatidae ) y Loxosceles devia (Sicariidae); por otro lado, hubo siete especies que solo aparecieron una sola vez en la muestra. La diversidad total encontrada para el interior fue baja, y se mantuvo más o menos constante para las diversas zonas de la ciudad. El Cuadro 2, resume los valores encontrados.

En el exterior de las casas, las especies más abundantes fueron Latrodectus geometricus y L. mactans (Theridiidae), y 14 especies solo aparecieron una vez en la muestra (Cuadro 4). Los valores de diversidad total para el exterior de las casas fué muy parecida a la del interior, pero varío ampliamente entre las zonas de la ciudad, siendo la zona sur la que presento menor diversidad, mientras que el oriente presentó los valores más altos (Cuadro 2). Esto se hace mas patente al comparar las diversidades de orden 1 (Fig. 2). Se puede ver que en el interior de las viviendas, los intervalos de confianza para las ${ }^{1} \mathrm{D}$ se sobreponen (Fig. 2A); mientras que para el exterior, no existe traslape entre el oriente y las restantes zonas (Fig. 2B). Comparando las ${ }^{1} \mathrm{D}$ para el exterior y el interior de las casas, encontramos que la diversidad dentro de las viviendas, y la asociada a la periferia de éstas, son muy semejantes (Fig. 2C).

El cálculo de la riqueza específica por medio de las curvas de acumulación de especies arrojó amplias diferencias entre los valores observados $\left(\mathrm{S}_{\text {int }}=30 ; \mathrm{S}_{\text {ext }}=37\right.$, cuadro 2) y los valores calculados mediante su ajuste a la

Cuadro 2. Índices obtenidos para los muestreos de araneidos del ambiente interior y exterior de las viviendas de Ciudad Victoria, Tamps. $\mathrm{H}=$ Diversidad de Shannon-Weaver, Var $(\mathrm{H})=$ Varianza de la Diversidad, $\mathrm{H}_{\max }=$ Diversidad Máxima; $\mathrm{J}=$ Equitatividad de Pielou, $\mathrm{N}_{\mathrm{i}}=\mathrm{Número}$ total de individuos colectados, $\mathrm{S}$ = Número de especies observadas.

\begin{tabular}{|c|c|c|c|c|c|c|c|}
\hline Sitio & Índice & $\mathrm{C}$ & $\mathrm{N}$ & S & $\mathrm{O}$ & $\mathrm{P}$ & $\mathrm{T}$ \\
\hline \multirow[t]{4}{*}{ Interior } & $\mathrm{H}$ & 2.6 & 2.66 & 2.74 & 2.91 & 2.99 & 2.86 \\
\hline & $\operatorname{Var}(\mathrm{H})$ & 0.231 & 0.0198 & 0.0193 & 0.0332 & 0.0347 & 0.011 \\
\hline & $\mathrm{J}$ & 0.666 & 0.638 & 0.657 & 0.663 & 0.681 & 0.584 \\
\hline & $\mathrm{N}_{\mathrm{i}}$ & 118 & 128 & 145 & 146 & 155 & 692 \\
\hline \multirow[t]{5}{*}{ Exterior } & $\mathrm{H}$ & 1.978 & 2.177 & 1.648 & 2.726 & 2.332 & 2.88 \\
\hline & $\operatorname{Var}(\mathrm{H})$ & 0.0658 & 0.0345 & 0.0485 & 0.0398 & 0.016 & 0.0241 \\
\hline & $\mathrm{H}_{\text {max }}$ & 3.17 & 3.807 & 4.524 & 3.807 & 4.585 & 5.17 \\
\hline & $\mathrm{J}$ & 0.624 & 0.572 & 0.364 & 0.716 & 0.509 & 0.557 \\
\hline & $N_{i}$ & 26 & 37 & 51 & 40 & 47 & 201 \\
\hline
\end{tabular}


Cuadro 3. Listado de especies de arañas colectadas en el interior de las viviendas de Ciudad Victoria, Tamps. C $=\mathrm{Z}$ (ona Centro, $\mathrm{N}=\mathrm{Zona}$ Norte, $\mathrm{S}=$ Zona Sur, $\mathrm{O}=$ Zona Oriente, $\mathrm{P}=$ Zona Poniente, $\mathrm{Ab}=$ Abundancia Total, $\mathrm{Ca}=$ Número de casas donde se localizó la especie, $\mathrm{M}=$ Número de meses en los que se colectó la especie.

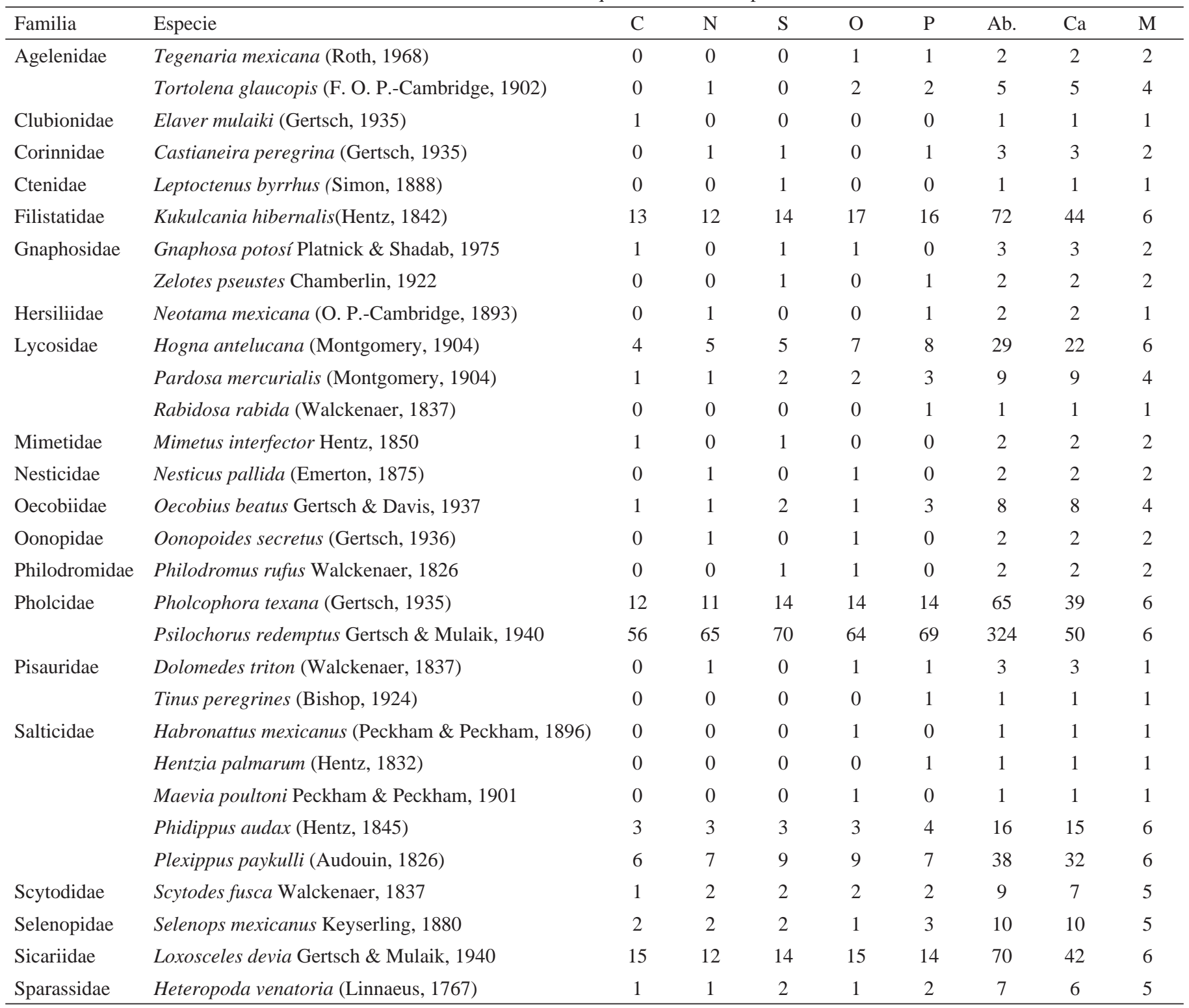

ecuación de Clench. Para especies dentro de las viviendas $\mathrm{S}_{\text {esp }}=(0.83 / 0.021)=39.5$ especies, mientras que para el exterior $\mathrm{S}_{\text {esp }}=(0.782 / 0.015)=52.1$ Las curvas de acumulación, así como sus respectivas ecuaciones de ajuste, para ambos ambientes, se presentan en la Fig. 3.

La caracterización de las especies encontradas como sinantrópicas, para el interior de la vivienda, arrojó resultados muy variables según el índice empleado; Mientras que los índices basados en densidad (ID) no permiten reconocer ninguna especie como común y solo una (Psilochorus redemptus), se considera frecuente, los índices basados en infestación (II) y permanencia (IP) reconocen 13 comunes, aunque solo están de acuerdo en 12 y difieren en una. El Índice de Infestación cataloga 13 especies comunes, 10 frecuentes y siete como ocasionales, mientras que el Índice de Permanencia (IP) clasifica 12 especies como comunes, nueve frecuentes y nueve ocasionales; hay que hacer notar que en la categoría "frecuentes" ambos índices concuerdan unicamente en ocho especies (ver Cuadro 5).

$\mathrm{Al}$ emplear el método gráfico O-T, se separan las especies en tres grupos (Fig. 4, Cuadro 5): Comunes, Frecuentes y Ocasionales. Las comunes comprenden, según este método, Psilochorus redemptus, Kukulcania hibernalis, Loxosceles devia, Pholcophora texana y Plexippus paykulli. 
Cuadro 4. Listado de especies de arañas colectadas en el exterior de las viviendas de Ciudad Victoria, Tamps. $\mathrm{C}=\mathrm{Zona}$ Centro, $\mathrm{N}=\mathrm{Zona}$ Norte, $\mathrm{S}=$ Zona Sur, $\mathrm{O}=$ Zona Oriente, $\mathrm{P}=$ Zona Poniente, $\mathrm{Ab}=$ Abundancia Total, $\mathrm{Ca}=$ Número de casas donde se localizó la especie, $\mathrm{M}=$ Número de meses en los que se colectó la especie.

\begin{tabular}{|c|c|c|c|c|c|c|c|c|c|}
\hline Familia & Especie & $\mathrm{C}$ & $\mathrm{N}$ & $\mathrm{S}$ & $\mathrm{O}$ & $\mathrm{P}$ & Ab. & $\mathrm{Ca}$ & M \\
\hline Dipluridae & Eagrus sp & 0 & 0 & 0 & 0 & 2 & 2 & 2 & 2 \\
\hline Theraphosidae & Aphonopelma sp & 0 & 1 & 1 & 0 & 1 & 3 & 3 & 2 \\
\hline \multirow{6}{*}{ Araneidae } & Eriophora edax (Blackwall, 1863) & 0 & 0 & 1 & 0 & 0 & 1 & 1 & 1 \\
\hline & Metepeira minima Gertsch, 1936 & 0 & 0 & 0 & 0 & 1 & 1 & 1 & 1 \\
\hline & Neoscona oaxacensis (Keyserling, 1864) & 2 & 4 & 2 & 3 & 4 & 15 & 15 & 6 \\
\hline & Gasteracanta cancriformes (Linnaeus, 1758) & 0 & 1 & 2 & 2 & 2 & 7 & 7 & 5 \\
\hline & Verrucosa arenata (Walckenaer, 1841) & 0 & 2 & 0 & 0 & 0 & 2 & 2 & 1 \\
\hline & Wagneriana tauricornis (O. P.-Cambridge, 1889) & 0 & 0 & 0 & 0 & 1 & 1 & 1 & 1 \\
\hline Oxyopidae & Peucetia viridians (Hentz, 1832) & 1 & 2 & 2 & 2 & 4 & 11 & 8 & 5 \\
\hline Pisauridae & Dolomedes triton (Walckenaer, 1837) & 0 & 1 & 0 & 0 & 1 & 2 & 2 & 2 \\
\hline \multirow[t]{3}{*}{ Salticidae } & Bagheera kiplingi (Peckham \& Peckham, 1896) & 0 & 0 & 1 & 0 & 0 & 1 & 1 & 1 \\
\hline & Phidippus audax (Hentz, 1845) & 0 & 0 & 2 & 0 & 0 & 2 & 2 & 2 \\
\hline & Plexippus paykulli (Audouin, 1826) & 0 & 0 & 2 & 0 & 0 & 2 & 2 & 2 \\
\hline Scytodidae & Scytodes fusca Walckenaer, 1837 & 0 & 0 & 1 & 0 & 0 & 1 & 1 & 1 \\
\hline Sicariidae & Loxosceles devia Gertsch \& Mulaik, 1940 & 1 & 2 & 1 & 3 & 1 & 8 & 8 & 5 \\
\hline Sparassidae & Heteropoda venatoria (Linnaeus, 1767) & 0 & 1 & 0 & 0 & 0 & 1 & 1 & 1 \\
\hline Tetragnathidae & Leucage argyra (Walckenaer, 1841). & 0 & 0 & 1 & 0 & 0 & 1 & 1 & 1 \\
\hline \multirow{3}{*}{ Theridiidae } & Latrodectus mactans (Fabricius, 1775) & 5 & 7 & 7 & 5 & 4 & 28 & 24 & 6 \\
\hline & Theridion australe (Banks, 1899) & 0 & 0 & 0 & 1 & 0 & 1 & 1 & 1 \\
\hline & Tidarren sisyphoides (Walckenaer, 1841) & 1 & 1 & 2 & 2 & 2 & 8 & 8 & 5 \\
\hline \multirow[t]{4}{*}{ Thomisidae } & Bassaniana versicolor (Keyserling, 1880) & 0 & 0 & 0 & 1 & 0 & 1 & 1 & 1 \\
\hline & Mecaphesa celer (Hentz, 1847) & 0 & 0 & 0 & 0 & 1 & 1 & 1 & 1 \\
\hline & Misumena vatia (Clerck, 1757) & 0 & 0 & 1 & 0 & 1 & 2 & 2 & 2 \\
\hline & Xysticus gulosus Keyserling, 1880 & 0 & 0 & 0 & 1 & 0 & 1 & 1 & 1 \\
\hline \multirow[t]{2}{*}{ Uloboridae } & Uloborus americanus Walckenaer, 1841 & 0 & 1 & 0 & 1 & 1 & 3 & 3 & 3 \\
\hline & Philoponella semiplumosa (Simon, 1893) & 0 & 0 & 0 & 0 & 1 & 1 & 1 & 1 \\
\hline
\end{tabular}

Para el exterior de las viviendas, dado que no se puede hablar de sinantropismo, se presentan los resultados como porcentajes de abundancia; dos especies representan el $43 \%$ de ocurrencias, Latrodectus geometricus con $29.81 \%$ y L. mactans, con $13.46 \%$; siete especies aparecieron en porcentajes entre $3 \%$ y $8 \%, 14$ entre $1 \%$ y $2 \%$ y 14 especies solo aparecieron una vez en la muestra, con $0.56 \%$ de ocurrencia; ocho especies, se colectaron tanto en el ambiente interior de las viviendas como en el exterior (Fig. 5).

Los Analísis de Similitud para la araneofauna colectada en el interior de las viviendas, mostraron valores al- 

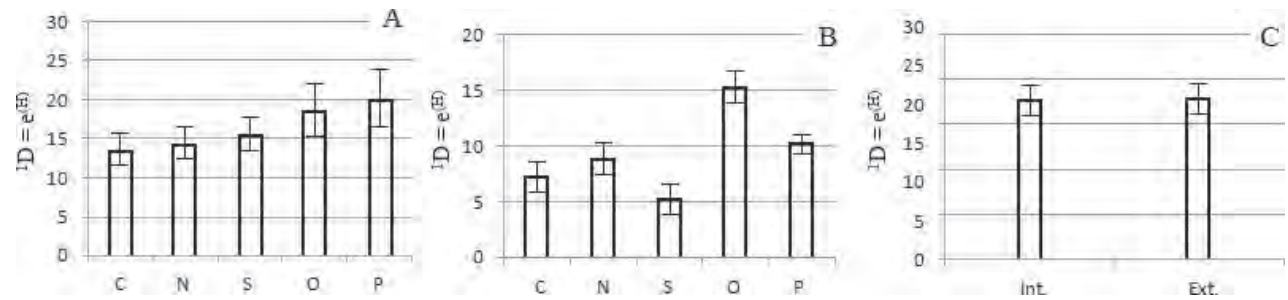

Figura 2. Diversidad de nivel 1 para la fauna de arañas de Ciudad Victoria, Tamps. A) Interior de las viviendas, B) Exterior de las viviendas, C) Total, interior y exterior. En las abscisas: $\mathrm{C}=$ Centro, $\mathrm{N}=$ Norte, $\mathrm{S}=$ Sur $\mathrm{O}=$ Oriente, $\mathrm{P}=$ Poniente.

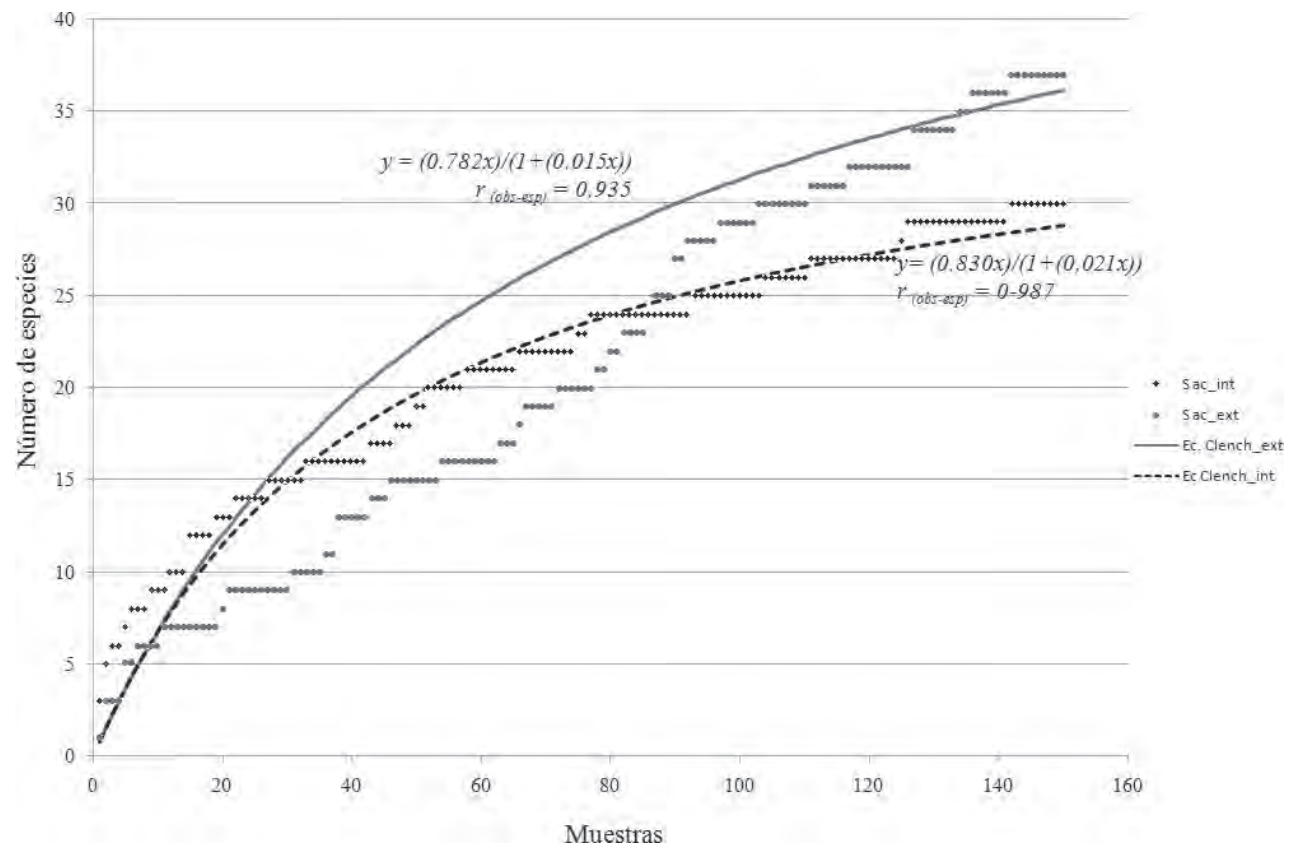

Figura 3. Curvas de acumulación de especies para las arañas colectadas en el interior y exterior las viviendas de Ciudad Victoria, Tamps. Las ecuaciones corresponden al Modelo de Clench, y los valores de regresión, al ajuste entre los valores observados y el modelo de Clench.

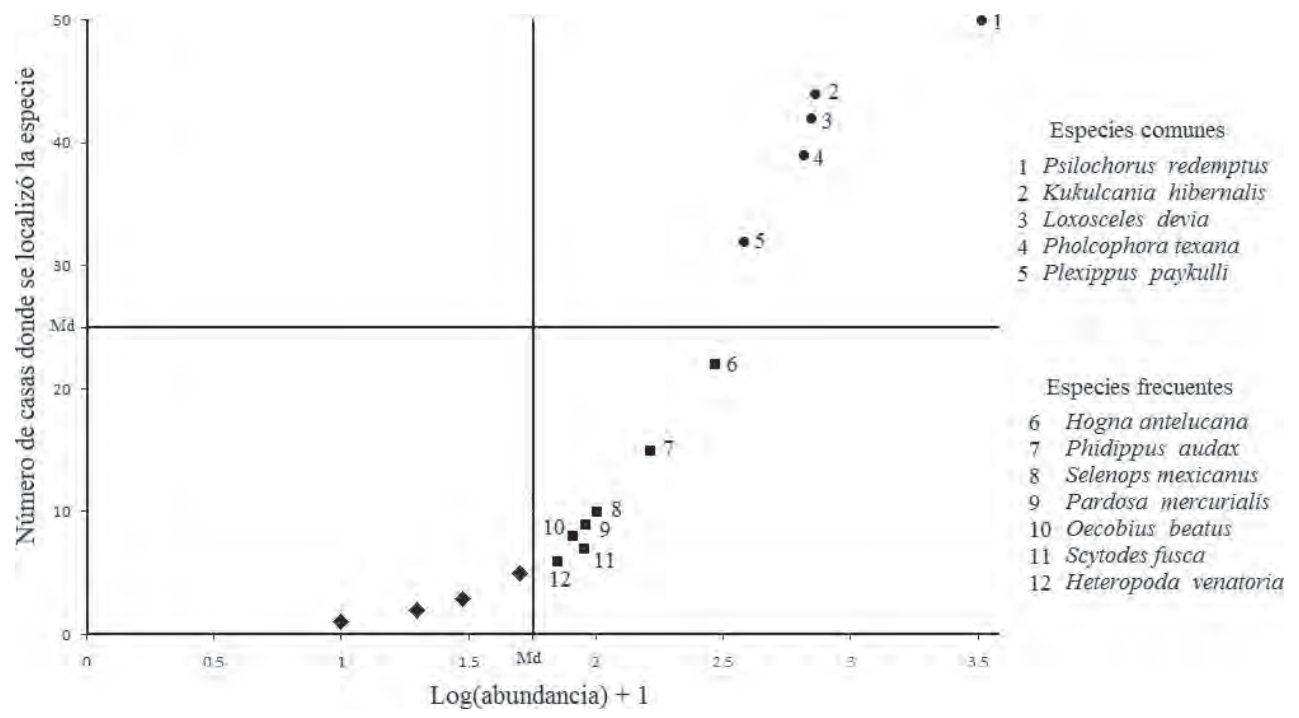

Figura 4. Gráfico de Olmstead-Tukey para los niveles de Sinantropismo de las arañas colectadas en el interior de las viviendas de Ciudad Victoria, Tamps. 
Cuadro 5. Niveles de Sinantropía de las especies de arañas de las viviendas de Ciudad Victoria, Tamps. ID = Índice de Densidad, NS ID $_{\text {I }}$ Nivel de Sinantropía basado en el Índice de Densidad, II = Índice de Infestación, NS II = Nivel de Sinantropía basado en el Índice de Infestación, IP

= Índice de Permanencia, $\mathrm{NS}_{\mathrm{IP}}=$ Nivel de Sinantropía basado en el Índice de Permanencia, O-T = Nivel de Sinantropía basado en el Índice de Olmstead-Tukey; $\mathrm{A}=$ Accidentales, $\mathrm{O}=$ Ocasionales, $\mathrm{F}=$ Frecuentes, $\mathrm{C}=$ Comunes.

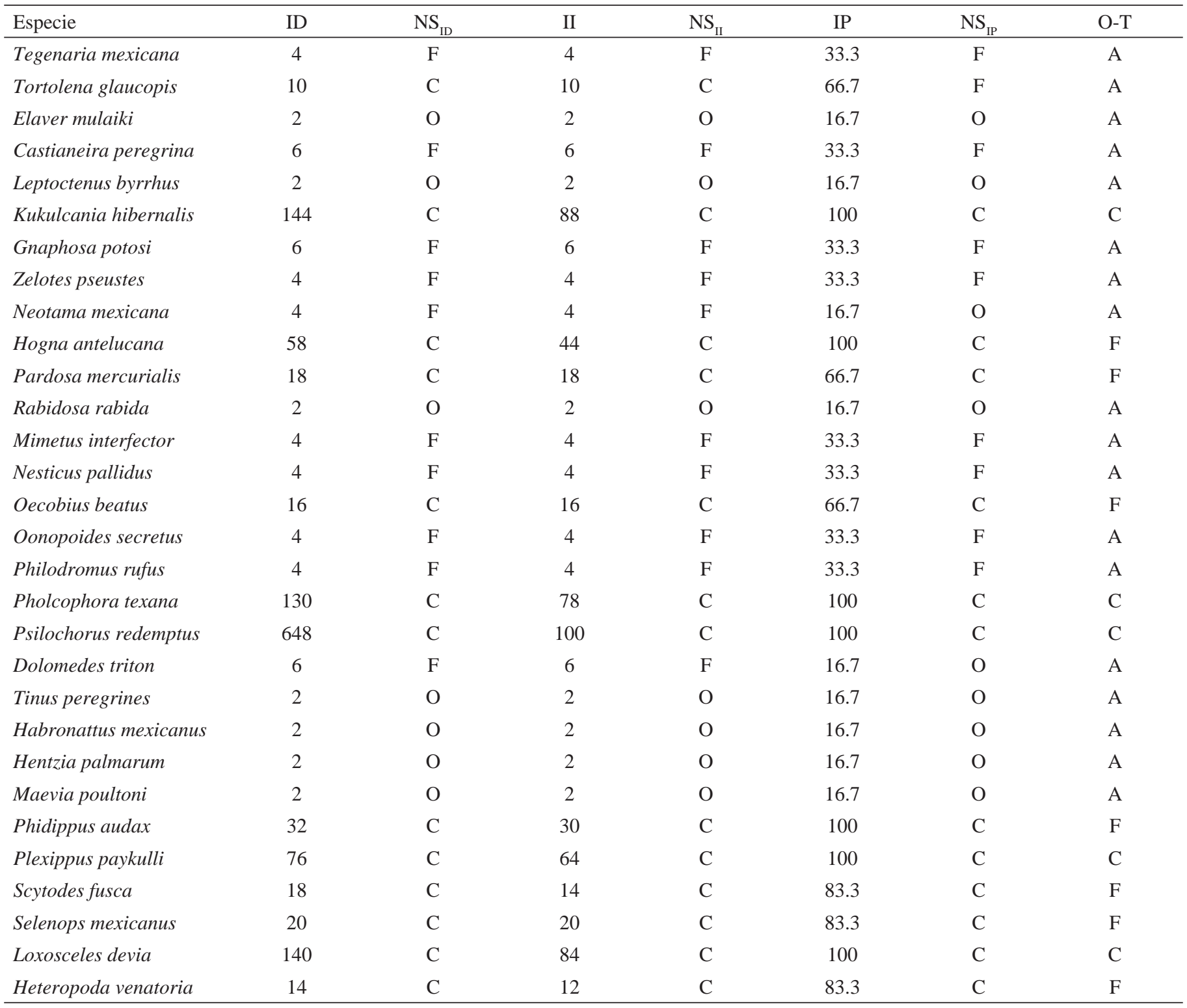

tos, presentando las zonas Centro y Sur una semejanza de 0.74 , mientras que las tres restantes, son similares a un nivel de 0.7 (Cuadro 6, Fig. 6). La similtud fuera de las viviendas, por otro lado, fue baja; el valor más alto se presentó entre la zona Centro y la zona Oriente, con un valor de 0.533 (Cuadro 7, Fig. 7).

\section{DISCUSIÓN}

Los resultados permiten reconocer las especies mas comunes en la zona urbana de Ciudad Victoria. Este dato es importante porque señala entre otras, a tres especies muy abundantes como habitantes comunes en el interior o en la periferia de las casas de los victorenses: Loxosceles devia, especie que se localizó en el interior de las viviendas y presentó la categoría de común en las clasificaciones de sinantropía, y Latrodectus geometricus y $L$. mactans, muy abundantes en la periferia de las viviendas, y a pesar de que no existen datos oficiales sobre aracnoidismo en el estado, estas especies son potencialmente peligrosas (Quintana Castillo \& Otero Patiño 2002; Goddard et al. 2008; Almeida et al. 2009). Entre las especies encontradas, dos de ellas son un primer registro para el estado: Elaver mulaiki (Clubionidae) y Bagheera kiplingi (Salticidae). La primera (Elaver mulaiki), es una araña de saco que se localizó en la pared de una ventana con gran 
Cuadro 6. Valores de Similitud de Jaccard para la fauna de arañas en el interior de las viviendas, entre las zonas de la ciudad. $\mathrm{C}=$ Centro, $\mathrm{N}=$ Norte, $\mathrm{S}=$ Sur, $\mathrm{O}=$ Oriente, $\mathrm{P}=$ Poniente.

\begin{tabular}{cccccc}
\hline & $\mathrm{C}$ & $\mathrm{N}$ & $\mathrm{S}$ & $\mathrm{O}$ & $\mathrm{P}$ \\
\hline $\mathrm{C}$ & 1 & 0.571 & 0.737 & 0.565 & 0.5 \\
$\mathrm{~N}$ & & 1 & 0.565 & 0.696 & 0.696 \\
$\mathrm{~S}$ & & & 1 & 0.56 & 0.56 \\
$\mathrm{O}$ & & & & 1 & 0.556 \\
$\mathrm{P}$ & & & & & 1 \\
\hline
\end{tabular}

Cuadro 7. Valores de Similitud de Jaccard para la fauna de arañas en el exterior de las viviendas, entre las zonas de la ciudad. $\mathrm{C}=$ Centro, $\mathrm{N}=$ Norte, $\mathrm{S}=$ Sur, $\mathrm{O}=$ Oriente, $\mathrm{P}=$ Poniente.

\begin{tabular}{cccccc}
\hline & $\mathrm{C}$ & $\mathrm{N}$ & $\mathrm{S}$ & $\mathrm{O}$ & $\mathrm{P}$ \\
\hline $\mathrm{C}$ & 1 & 0.438 & 0.391 & 0.533 & 0.375 \\
$\mathrm{~N}$ & & 1 & 0.423 & 0.474 & 0.407 \\
$\mathrm{~S}$ & & & 1 & 0.321 & 0.516 \\
$\mathrm{O}$ & & & & 1 & 0.407 \\
$\mathrm{P}$ & & & & & 1 \\
\hline
\end{tabular}

cantidad de residuos de construcción; Bagheera kiplingi se colectó de un jardín que contenia suelo con humus que se habia obtenido de un vivero.

No parece existir diferencia entre la diversidad total del interior de la vivienda y el exterior, aunque su similitud es muy baja $(S \mathrm{j}=0.13)$, lo que significa que aunque ambos ambientes presentan una sólida estructura de especies, estas estructuras presentan una composición diferente. Por otro lado, la diversidad en el interior de las viviendas se mantiene alta y constante, mientras que en el exterior es menor y varía de una zona a otra, lo que lo atribuimos al ambiente interior, más homogéneo y el exterior de las casas, más variado y transitorio, atribuible principalmente a la vegetación ornamental.

Los valores esperados de especies mediante el ajuste de Clench para las Curvas de Acumulación de Especies parecen apoyar esto último; a pesar de que ambas curvas presentan un buen ajuste con los valores observados, la curva para interiores parece acercarse a una asíntota más rápidamente que la curva para el exterior, lo que nos lleva a suponer que el muestreo para interiores, resultó más efectivo debido a la mayor homogeneidad del ambiente interno, mientras que la curva relativa al muestreo externo continúa creciendo debido a la mayor heterogeneidad del ambiente y precisa un esfuerzo mayor de muestreo.

Los resultados para sinantropía obtenidos de los índices numéricos mostraron congruencia entre sí; los tres índices (ID, II e IP) categorizan 12 especies como sinantrópicas comunes: Tortolena glaucopis, Kukulcania hibernalis, Hogna antelucana, Pardosa mercurialis,
Oecobius beatus, Pholcophora texana, Psilochorus redemptus, Phidippus audax, Plexippus paykulli, Scytodes fusca, Selenops mexicanus, Loxosceles devia, y Heteropoda venatoria. Ocho especies son consideradas como frecuentes en las viviendas de Ciudad Victoria: Tegenaria mexicana, Castianeira peregrina, Gnaphosa potosí, Zelotes pseustes, Mimetus interfector, Nesticus pallidus, Oonopoides secretus y Philodromus rufus. De las restantes diez especies, siete de ellas se consideran como ocasionales por los tres índices y solo en tres especies, existe discrepancia en cuanto a la categorización. Por otro lado, el índice O-T fue más conservador en la calificación de sinantropía; de las 12 especies consideradas por los índices numéricos como comunes, O-T solo otorga el calificativo de "sinantrópico común" a Psilochorus redemptus, Kukulcania hibernalis, Loxosceles devia, Pholcophora texana y Plexippus paykulli, mientras que a las siete especies restantes calificadas como comunes por ID, II e IP, $\mathrm{O}-\mathrm{T}$ las etiqueta como "sinantrópicas frecuentes". Esto nos lleva a concluir que el índice O-T es más estricto para calificar la sinantropía, mientras los anteriores parecen ser más permisivos. La relación gráfica entre el logaritmo de la abundancia y el número de viviendas en los cuales aparece un taxón, parecen ser más sensibles que los índices basados únicamente en conteos; ejemplo de esto es el caso de Hogna antelucana y Phidippus audax, que clasificados como “comunes” por los índices numéricos, O-T los califica únicamente como "frecuentes", o el caso de Tortolena glaucopis, categorizada como “común” por ID e II, por el hecho de haber sido localizada en el 10\% de las casas; y como "frecuente" por IP, por haber aparecido durante cuatro meses, O-T no la coloca entre las relación por su baja abundancia en la muestra.

La similitud para el interior de las viviendas fue alto con muy ligeras diferencias entre las zonas urbanas; El centro de la ciudad (C) y el Sur (S) son las zonas con mayor semejanza en la composición de especies, lo que puede explicarse por ser las partes mas antiguas de la ciudad, y por compartir las riberas del Río Corona, que la atraviesa, aunque el resto de área urbana no difiere mayormente de éstas. Para el exterior, la similitud entre las zonas de la ciudad, es más baja en general, lo que señala que las zonas comparten pocas especies entre sí; la semejanza más alta se da entre centro $(\mathrm{C})$ y oriente $(\mathrm{O})$, dado que el total de especies localizadas en C están presentes en O, pero ésta tiene casi un 50\% más de especies, lo que explica que la similitud sea baja. Atribuimos la poca semejanza entre las zonas de la ciudad a las diferencias en las áreas adyacentes que la rodean y la nutren de especies no sinantrópicas: hacia el Oriente de Ciudad Victoria, se presenta baja diversidad de vegetación debido a los trabajos de expansión urbana; el Sur y el Poniente bordean la 


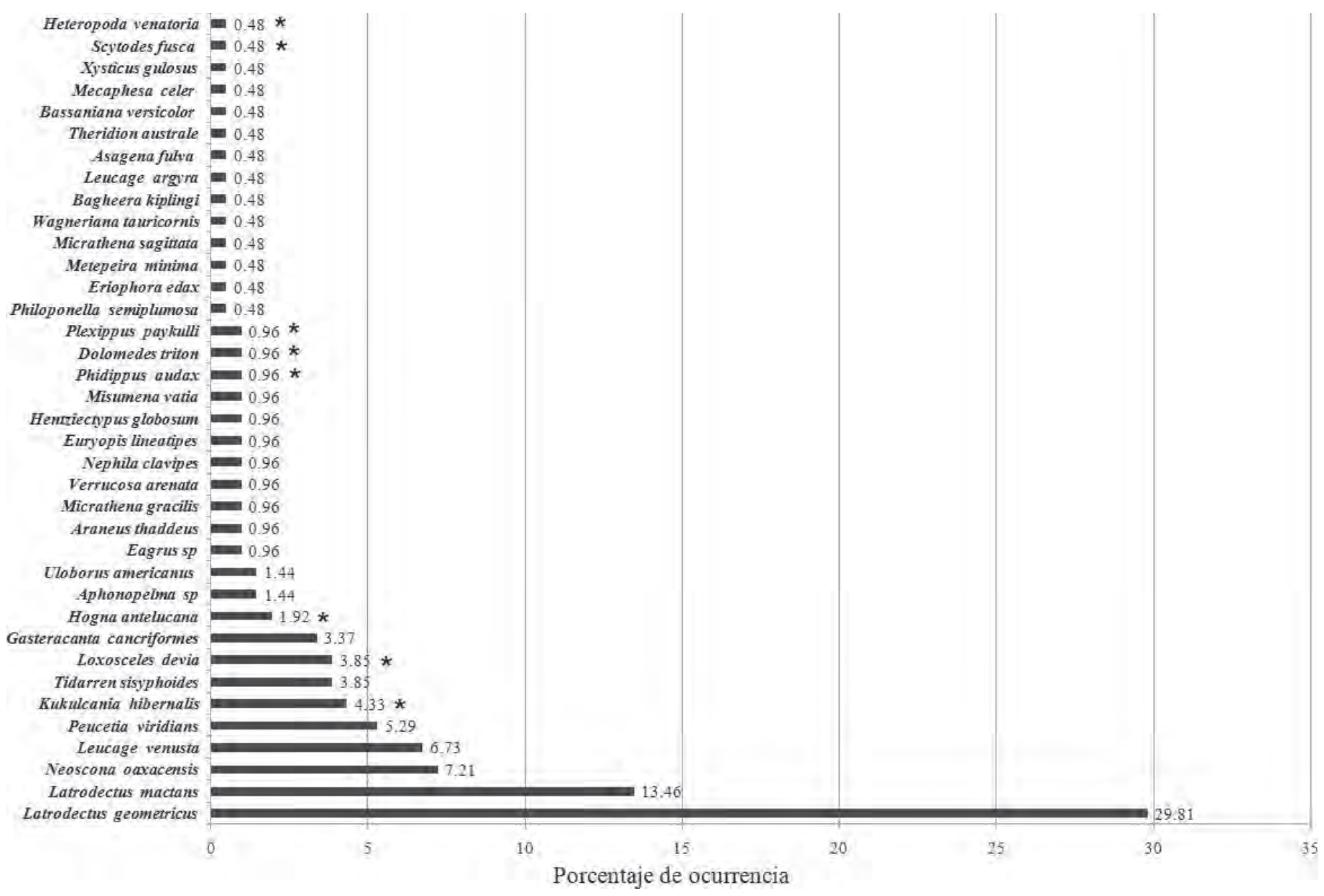

Figura 5. Especies encontradas y sus porcentajes de ocurrencia fuera de las viviendas de Ciudad Victoria. Las especies marcadas (*) mostraron importante presencia en el interior de las casas muestreadas

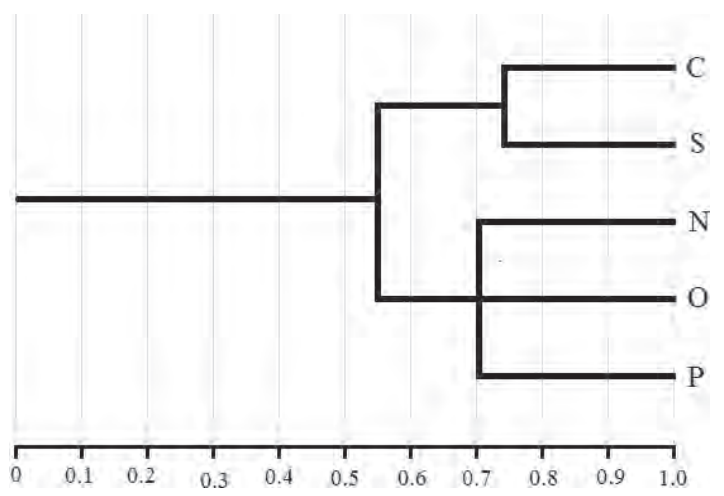

Figura 6. Gráfico de similitud para el interior de las viviendas de Ciudad Victoria. C, Zona Centro; S, Sur; N, Norte, O, Oriente; P, Poniente.

Sierra que presenta vegetación submontana, aunque el sur presenta alto disturbio, mientras que el norte es una zona con lomeríos bajos bordeda por matorrales naturales de poco tamaño y actividad agrícola, que la diferencía de las zonas restantes.

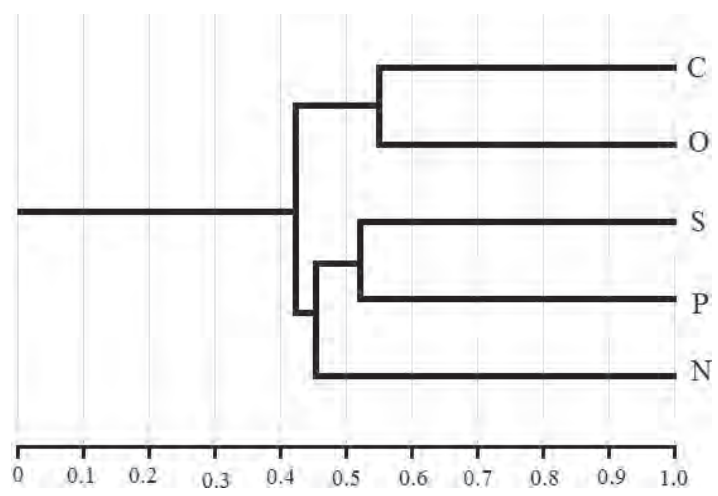

Figura 7. Gráfico de similitud para el exterior de las viviendas de Ciudad Victoria. C, Zona Centro; O, Oriente; S, Sur; P, Poniente; N, Norte.

Agradecimientos. El primer autor desea agradecer a la Biol. MC Ma. Concepción Herrera Monsivais por la lectura crítica del manuscrito original y sus valiosas opiniones. Hacemos patente nuestro agradecimiento a los revisores de este trabajo por sus observaciones y sugerencias, y a todas y cada una de las personas que ayudaron a la realización de este trabajo. 


\section{LITERATURA CITADA}

Almeida R., Ferreira Junior, R. S., Chaves, C. R. \& Barraviera, B. 2009. Envenomation caused by Latrodectus geometricus in São Paulo state, Brazil: a case report. Journal of Venomous Animals and Toxins including Tropical Disease [online], 15: 562-571.

Anónimo. 1983. Síntesis Geográfica del Estado de Tamaulipas. Secretaría de Programación y Presupuesto. Instituto Nacional de Estadística, Geografía e Informática. MX. 158 pp.

Borda, C. E., Delgado, M. S. \& Rea, M. J. F. 2005. Arácnidos de importancia médica de las especies Loxosceles en la región de influencia de la Universidad Nacional del Nordeste. Universidad Nacional del Nordeste, Comunicaciones Científicas y Tecnológicas, Resumen m-141. Corrientes, AR. 4 pp.

Bradley, R. E. 2013. Common Spiders of North America. University of California Press, Berkeley, USA. 280 pp.

Brady A. R. 1964. The Lynx spiders of north America, North of Mexico (Araneae, Oxyopidae), Bulletin of the Museum of Comparative Zoology, 131: 429-518.

Brower, J. E. \& Zar, J. H. 1977. Field and Laboratory Methods for General Ecology. WMC Brown Company Publishers, Dubuque, Iowa, US. 185 pp.

Castelo, J. L. \& Pérez, T. M. 1999. El problema en la determinación taxonómica de las arañas Salticidae (Arachnida: Araneae) habitantes de viviendas de la Ciudad de México. Memorias del XXXIV Congreso Nacional de Entomología, Sociedad Mexicana de Entomología. Aguascalientes, Aguascalientes, pp. 33-37.

Chickering, A. M. 1937. Notes and studies on Arachnida, III. Arachnida from the San Carlos Mountains. pp. 271-283. In: L. B. Kellum, E. H. Watson, E. S. Bastin, R. W. Imlay, L. R. Dice, A. M. Chickering, R. V. Chamberlin, C. L. Hubbs, H. T. Gaige (Eds.). The Geology and Biology of the San Carlos Mountains, Tamaulipas, Mexico. University of Michigan Studies: Scientific Series, XII. Ann Arbor University of Michigan Press, USA.

Coldwell, R. K. 2013. EstimateS: Statistical estimation of species richness and share species from samples. Version 9. On line at: purl.oclc.org/estimates.

Cupul-Magaña, F. G \& Navarrete Heredia, J. L. 2008. Artropodofauna de las viviendas de Puerto Vallarta, Jalisco, México. Ecología Aplicada, 7: 187-190.

Desales-Lara, M. A. 2013. Descripción de una nueva especie del género Filistatinella Gertsch \& Ivie, 1936 (Araneae: Filistatidae) de Toluca, México. Revista Ibérica de Aracnología, 21: 51-55.

Desales-Lara, M. A., Francke, O. F. \& Sánchez-Nava, P. 2011. Arañas (Arachnida: Araneae) asociadas a diferentes grados de urbanización. Memorias del XLVI Congreso Nacional de Entomología, Cancún-Riviera Maya, Quintana Roo, pp. 69-73.

Desales-Lara, M. A., Francke, O. F. \& Sanchez-Nava, P. 2013. Diversidad de arañas (Arachnida: Araneae) en hábitats antropogénicos. Revista Mexicana de Biodiversidad, 84: 291-305.

Durán-Barron, C. G. 2007. El sinantropismo en arañas. Memorias del VII Congreso Latinoamericano de Entomología y XLII Congreso Nacional de Entomología. Sociedad Mexicana de Entomología. Acapulco, Guerrero, pp. 9-13.

Durán-Barrón, C. G. \& Pérez-Ortiz , T. M. 2005. Diversidad de familias de arañas (Arachnida: Araneae) asociadas a viviendas humanas de la Ciudad de México. Memorias del XL Congreso Nacional de Entomología, Sociedad Mexicana de Entomología. Tapachula, Chiapas. 66-70.

Durán-Barrón, C. G., Francke, O. F. \& Pérez-Ortiz, T. M. 2009. Diversidad de arañas (Arachnida: Araneae) asociadas a viviendas de la ciudad de México (Área metropolitana). Revista Mexicana de Biodiversidad, 80: 55-69.

Gertsch, W. J. \& Davis, L. I. 1937. Report on a Collection of Spiders from Mexico. I. American Museum Novitates, 961: 29 pp.

Gertsch W. J. \& Davis, L. I. 1940. Report on a Collection of Spiders from Mexico. II. American Museum Novitates, 1059: 18 pp.

Gertsch W. J. \& Davis, L. I. 1940. Report on a Collection of Spiders from Mexico. III. American Museum Novitates, 1069: 22 pp.

Gertsch W. J. \& Davis, L. I. 1942. Report on a Collection of Spiders from Mexico. IV. American Museum Novitates, 1158: 19 pp.

Gertsch W. J. \& Davis, L. I. 1946. Report on a Collection of Spiders from Mexico. V. American Museum Novitates, 1313: 11 pp.

Gertsch, W. J. \& Ennik, F. 1983. The spider genus Loxosceles in North America, Central America, and the West Indies (Araneae, Loxoscelidae). Bulletin American Museum of Natural History, 175: 264-360.

Gertsch W. J. \& Mulaik, S. 1940. The spiders of Texas. Bulletin American Museum of Natural History, 77: 307-340.

Goddard, J., Upshaw, S., Held, D. \& Johnson, K. 2008. Severe reaction from envenomation by the brown widow spider, Latrodectus geometricus (Araneae: Theridiidae). Southern Medical Journal, 101: 1269-1270.

Gómez-Rodríguez, J. F. \& Salazar-Olivo, C. A. 2012. Arañas de la región montañosa de Miquihuana, Tamaulipas: listado faunístico y registros nuevos. Dugesiana, 19: 1-7.

Gómez-Rodríguez, J. F., Montaño, H., Ibarra-Nuñez, G. \& Salazar-Olivo, C. A. (en prensa). Arácnidos (excepto ácaros) de Tamaulipas: listado actualizado y algunos registros nuevos. In: Correa-Sandoval, A. (Ed.). Biodiversidad Tamaulipeca, Vol 2. Dirección General de Educación Tecnológica - Instituto Tecnológico de Ciudad Victoria, México.

Hoffmann, A. 1976. Relación bibliográfica preliminar de las arañas de México (Arachnida: Araneae). Publicaciones especiales No. 3, Instituto de Biología. Universidad Nacional Autónoma de México. 177 pp.

Huber, B. A. 2000. New World pholcid spiders (Araneae: Pholcidae): A revision at generic level. Bulletin of American Museum of Natural History, 254: 1-348.

INEGI, 2012. Censo de Población y vivienda 2010, En linea en http:// www.inegi.org.mx/est/contenidos/proyectos/ccpv/cpv2010/

Jiménez, M. L. 1996. Capítulo 11, Araneae, pp. 82-101. In: J. Llorente, A.N. García \& E. González (Eds.) Biodiversidad, taxonomía y biogeografía de Artrópodos en México: hacia una síntesis de su conocimiento. Instituto de Biología, Universidad Nacional Autónoma de México, México.

Jiménez, M. L. 1998. Aracnofauna asociada a las viviendas de la ciudad de la Paz, B.C.S., México. Folia Entomológica Mexicana, 102: 1-10.

Jiménez-Valverde, A. \& Hortal, J. 2003. Las curvas de acumulación de especies y la necesidad de evaluar la calidad de los inventarios biológicos. Revista Ibérica de Aracnología, 8: 151-161.

Jocqué, R. \& Dippenaar-Schoeman, A. S. 2006. Spiders families of the world. Musée Royal de l'Afrique Centrale, Tervuren, BE. 336 pp.

Kaston, B. 1978. How to Know the Spiders. $3^{\text {rd }}$ ed. (Pictured Key Nature Series). Wm. C. Brown Company Publishers, Dubuque, Iowa.

Magurran, A. E. 2004. Measuring Biological Diversity. Wiley-Blackwell. 264 pp.

McIntyre, N E. 2000. Ecology of Urban Arthropods: A Review and a Call to Action. Annals of the Entomological Society of America, 93: 825-835. 
Moreno, C. E. 2001. Métodos para medir la biodiversidad. M\&T S. E. A. Manuales y Tesis, Sociedad Entomológica Aragonesa, vol. 1. Zaragoza, ES. 84 pp.

Platnick, N. I. 2014. The world spider catalog, version 14.0. American Museum of Natural History, online at http://research.amnh. org/entomology/spiders/catalog/index.html.

Prószyński, J. 2013. Introduction to classification of Salticidae. online at http://www.peckhamia.com/salticidae/Subfamilies/0-Classification.html.

Sneath, P. H. A. \& Sokal, R. R. 1973. Numerical Taxonomy, the Principles and Practice of Numerical Classification. W. H. Freeman and Company, San Francisco, USA. 573 pp.

Quintana-Castillo, J. C. \& Otero Patiño, R. 2002. Envenenamiento aracnídico en las Américas. MedUNAB, Universidad Autónoma de Bucaramanga, Colombia, 13: $1-9$.

Levi, H. W. 1965. Techniques for the study of spider genitalia. Psyche, 72: 152-158.
Levi, H. W. 2002. Keys to the Genera of Araneid Orb-weavers (Araneae, Araneidae) of the Americas. The Journal of Arachnology, 30: 527-562.

Levi H. W. \& Randolph, D. E. 1975. A Key and checklist of American spiders of the Family Theridiidae north of Mexico (Araneae). The Journal of Arachnology, 3: 31-51.

Reddell J. R. \& Elliott, W. R. 1973. A Checklist of the Cave Fauna of Mexico. IV. Additional Records from the Sierra de el Abra, Tamaulipas and San Luis Potosí. Bulletin of the Association for Mexican Cave Studies, 5: 171-180.

Ruiz-Cancino, E. \& Coronado-Blanco, J. M. 2002. Artrópodos Terrestres de los estados de Tamaulipas y Nuevo León, México. Centro de Investigación y Desarrollo Agropecuario, Forestal y de la Fauna - Universidad Autónoma de Tamaulipas, Serie Publicaciones Científicas, No. 4. Cd Victoria, Tamaulipas, México. 377 pp.

Ubick, D., Paquin, P., Cushing, P. E. \& Roth, V. (Eds.) 2005. Spiders of North America: An Identification Manual. American Arachnology Society. $377 \mathrm{pp}$. 